\title{
Linx
}

Revue des linguistes de l'université Paris X Nanterre

$7 \mid 1995$

Saussure aujourd'hui

\section{Louis Hjelmslev le véritable continuateur de}

Saussure

Kenji Tatsukawa

\section{(2) OpenEdition}

\section{Journals}

Édition électronique

URL : http://journals.openedition.org/linx/1241

DOI : 10.4000/linx.1241

ISSN : 2118-9692

\section{Éditeur}

Presses universitaires de Paris Nanterre

\section{Édition imprimée}

Date de publication : 1 septembre 1995

Pagination : 479-487

ISSN : 0246-8743

\section{Référence électronique}

Kenji Tatsukawa, «Louis Hjelmslev le véritable continuateur de Saussure », Linx [En ligne], 7| 1995,

mis en ligne le 24 juillet 2012, consulté le 19 avril 2019. URL : http://journals.openedition.org/linx/1241 ; DOI : 10.4000/linx.1241

Ce document a été généré automatiquement le 19 avril 2019.

Département de Sciences du langage, Université Paris Ouest 


\title{
Louis Hjelmslev le véritable continuateur de Saussure
}

\author{
Kenji Tatsukawa
}

Il est vrai qu'on trouve le nom de Louis Hjelmslev partout dans les écrits linguistiques et sémiotiques même s'il est moins fréquent que le nom de Saussure. Mais ce Hjelmslev, $\mathrm{n}$ est-il pas celui qui aurait déjà terminé sa modeste carrière scientifique dans l'histoire du structuralisme linguistique ? Ne pourrait-on pas dire que le « cœur de la potentialité » de la théorie hjelmslévienne du langage n'a pas été suffisamment lu jusqu'à aujourd'hui ? Ce que je me propose de développer dans ce qui suit, c'est que Hjelmslev, successeur et continuateur le plus direct de Saussure et théoricien du langage potentiellement le plus actuel, n'est pas du tout connu jusqu'à aujourd'hui.

\section{Saussure et le structuralisme immanentiste}

2 Pour Hjelmslev, qui se veut fondateur d'une linguistique radicalement nouvelle (la glossématique) en rupture avec le passé, Saussure est son « seul devancier », et Hjelmslev lui-même se considère comme le seul continuateur du maître genevois. Il est inutile de dire que Saussure ; du moins le Cours de Saussure, a influencé de nombreux linguistes, et par là a donné lieu à la naissance du structuralisme; mais en ce qui concerne la continuation et le développement aussi bien du structuralisme que de l'immanentisme, ainsi que des réflexions sur le sujet parlant chez Saussure, il me semble qu'il n'y a que Hjelmslev qui mérite le nom de véritable continuateur de Saussure.

3 Tout le monde sera d'accord pour dire que le caractère fondamental de la théorie hjelmslévienne du langage est le formalisme. Or, ce formalisme de Hjelmslev semble une belle synthèse de deux principes introduits par Saussure : d'une part le structuralisme, qui se fonde sur les distinctions langue / parole et synchronie / diachronie; et d'autre part l'immanentisme, lié à la distinction forme / substance (bien que cette terminologie ne soit pas propre à Saussure). Les structuralistes sont nombreux en linguistique, mais on trouve difficilement des linguistes dont la théorie incarne à la fois ces deux principes 
saussuriens. Or, le linguiste danois est sans doute le seul continuateur direct de ces deux principes saussuriens : c'est sur quoi je m'appuie pour dire que la théorie hjelmslévienne est un structuralisme immanentiste. L'immanentisme consiste à étudier les langues uniquement du point de vue « linguistique », c'est-à-dire du point de vue de la forme ou des relations intra-linguistiques sans faire recours à la "substance ", donc aux concepts donnés par d'autres sciences que la linguistique: philosophie, logique, psychologie, sociologie, etc. $»^{1}$

On sait que le linguiste danois a substitué aux signifiant / signifié chez Saussure la terminologie expression / contenu, et il distingue dans chacun de ces deux plans le niveau de la forme et celui de la substance pour privilégier bien entendu la description de la forme. Autrement dit, l'objet privilégié de la linguistique n'est autre que la forme en tant que réseau de relations intralinguistiques, ou la structure définie comme une "entité autonome de dépendances internes ${ }^{2} »$. La langue ainsi formalisée engloberait non seulement les langues au sens général du terme, c'est-à-dire les langues naturelles, mais aussi toute espèce de système sémiotique. Par conséquent, la théorie linguistique fondée sur le structuralisme immanentiste, qui se veut à tout prix intra-linguistique, devient paradoxalement la sémiologie, qui n'est rien d'autre que la trans-linguistique.

\section{Le dynamisme du langage}

5 Si la synchronie, objet primaire de la linguistique saussurienne, est un système statique, le dynamisme du langage est exclu de ce système synchronique pour se trouver à l'extérieur en tant que diachronie ${ }^{3}$. Par contre, pour Hjelmslev la synchronie de la langue est elle-même dynamique :

Il y a des causalités qui sont propres à la synchronie, des forces agissantes qui ne se reflètent pas immédiatement dans la diachronie, mais dont la vitalité, n'est pas pour cela moins évidente. Le synchronique n'est rien que la langue en fonctionnement, le jeu des oppositions entre signes. Le synchronique est une

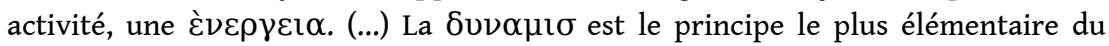
langage 4 .

6 Pour Hjelmslev, le système linguistique ou la forme n'est rien d'autre que le champ de la «force de relations" où les signes et les catégories s'appellent et se transforment mutuellement ${ }^{5}$. Il est important de remarquer que $1^{\circ}$ les différences dans le système synchronique chez Saussure sont toutes statiques, tandis que les oppositions chez Hjelmslev constituent des processus dynamiques entre signes ou entre catégories et ne sont jamais les conséquences statiques de ces processus; $2^{\circ} \mathrm{Hjelmslev}$ retrouve à l'intérieur même du système synchronique de la langue le dynamisme du langage, que son prédécesseur genevois a évacué en dehors de la synchronie; je pars de cette conception hjelmslévienne du langage pour dire qu'il est possible de formaliser le dynamisme du langage.

\section{Le sujet e(s)t la langue}

$7 \mathrm{Au}$ moment de la fondation de sa théorie linguistique, Saussure a fait recours au « sujet parlant ", qui est selon moi le sujet entendant, pour en faire le point de vue privilégié, c'est-à-dire le critère épistémologique de la linguistique ${ }^{6}$. Quant à Hjelmslev, il semblerait qu'il a exclu de la linguistique toute réflexion sur le « sujet parlant ainsi que sur la parole, 
acte de langage du sujet parlant. Il est vrai que le premier Hjelmslev n'est pas aussi rigoureux en disant dans les Principes de grammaire générale (1928) que la linguistique fait partie de la psychologie et que la langue est une réalité psychologique qui est de nature subconsciente.

Mais, déjà dans un ouvrage extrêmement important mais méconnu, La catégorie des cas (1935-37), le recours au sentiment linguistique du sujet est catégoriquement écarté en tant que procédé « psychologiste " par excellence, ce qui va conduire son auteur, avec son collaborateur Hans Jørgen Uldall, à la fondation de la glossématique, théorie pour laquelle la langue ne sera autre qu'une " réalité objective ».

Il y a pourtant dans ce texte un revirement très intéressant car les relations d'espace exprimées par la catégorie grammaticale de cas, telles que devant et derrière, à droite et à gauche ou " prés » et « loin », sont en rapport étroit avec le facteur sujet.

Le phénomène désigné par le signe linguistique $n$ est pas d'ordre objectif mais d'ordre subjectif. Le sujet parlant ne choisit pas les formes grammaticales selon les exigences de l'état de choses objectif ou réel, mais selon un principe imposé par la conception ou l'idée (Anschauung oder Idee) par laquelle il regarde le fait objectif. (...) Les significations fondamentales des cas et des prépositions recouvrent une seule et même catégorie conceptionnelle. Le phénomène subjectif désigné par cette catégorie est la conception spatiale; cette conception est appliquée par le sujet parlant aux divers ordres du phénomène objectif, qu'il s'agisse de l'espace, du temps, de la causalité logique ou de la rection syntagmatique ${ }^{7}$.

Si le « sujet parlant » n'était pour Saussure que le critère épistémologique pour construire des objets de la linguistique, Hjelmslev, lui, en transformant la langue saussurienne ou la conception saussurienne du système en quelque chose de plus souple et de plus complexe, arrive en quelque sorte à intérioriser ce sujet dans l'objet même de la linguistique qu'est la langue. En d'autres termes, ce que Hjelmslev se propose de saisir, ce n'est pas le sujet séparé du langage ni l'acte de discours jeté en dehors de la langue, mais c'est justement le sujet en tant que langue. Le sujet parlant se métamorphose ainsi en un constituant essentiel de l'objet même de la théorie du langage.

\section{La « sublogique » des langues naturelles}

11 C'est la théorie spécifiquement hjelmslévienne des oppositions dites participatives, développée entre autres dans $L a$ catégorie des cas, qui a permis de réaliser cette nouvelle articulation du sujet et du langage.

Selon Hjelmslev, la langue n'est pas un pur système logicomathématique tel qu'il est conçu par des structuralistes ordinaires, mais un système sublogique, c'est-à-dire à la fois logicomathématique et prélogique. Le terme "prélogique » est emprunté au sociologue français Lucien Lévy-Bruhl dont la recherche sur la mentalité des peuples dit primitifs est une source d'inspiration importante pour le linguiste danois. Cette nouvelle conception du système linguistique implique un changement d'optique décisif sur les oppositions. Dire que la langue n'est pas un système logicomathématique mais un système sublogique c'est dire justement que les oppositions constituant le système ne sont pas des oppositions binaires et exclusives de type $A$ / non- $A$, telles qu'elles sont conçues par Jakobson et ses partisans. Dans la langue en tant que système sublogique. il ne s'agit pas d'une opposition entre un terme positif et un terme négatif, mais il s'agit plutôt d'une opposition entre un terme précis et un terme vague qui engloberait le premier. 
Le système n'est pas construit comme un système logico-mathématique d'oppositions entre termes positifs et négatifs. Le système linguistique est libre par rapport au système logique qui lui correspond. Il peut être orienté différemment sur l'axe du système logique, et les oppositions qu'il contracte sont soumises à la loi de participation: il $\mathrm{n}$ y a pas d'opposition entre $\mathrm{A}$ et non-A, il n'y a que des oppositions entre A d'un côté et $\mathrm{A}+$ non- $\mathrm{A}$ de l'autre. La découverte $\mathrm{n}$ a rien de surprenant puisqu'on sait par les recherches de M. Lévy-Bruhl que le langage porte l'empreinte d'une mentalité prélogique ${ }^{8}$.

13 Hjelmslev appelle les termes précis (A) intensifs $(\alpha, \beta, \gamma)$ et les termes vagues $(A+$ nonA) extensifs $(\mathrm{A}, \mathrm{B}, \Gamma)$; et il propose trois types d'opposition participative comme dans le schéma suivant pour calculer par là les types de structures possibles par la combinaison de ces trois types d'opposition :

$\alpha$

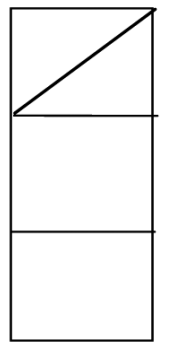

A

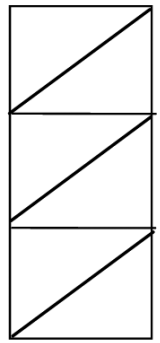

$\beta$

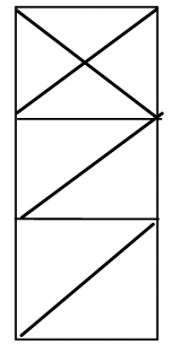

B

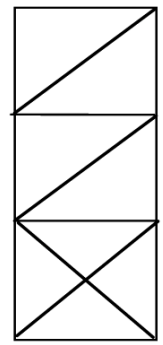

$\gamma$

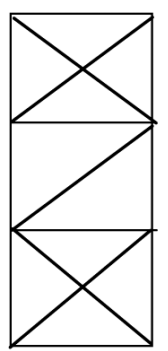

$\Gamma$

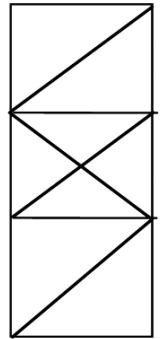

Le système hjelmslévien est ainsi pourvu d'une structure extrêmement souple qui n'exclut pas la contradiction logique : il s'agit d'un univers de la sublogique dans lequel le " grand » englobe le " petit », le « clair » englobe l'« obscur », le « masculin » englobe le "féminin », etc. A la différence de la théorie jakobsonienne des oppositions binaires qui est fondée uniquement sur les oppositions exclusives de type A / non-A, la théorie hjelmslévienne des oppositions participatives semble jeter une nouvelle lumière sur la logique des largues naturelles; on voit que celle-ci est complètement différente de la logique formelle classique, de telle sorte qu'elle permet d'analyser et de décrire scientifiquement le champ de la subjectivité longtemps négligée par la linguistique.

Selon l'article célèbre de Freud sur le sens opposé des mots originaires $(1910)^{9}$, les langues primitives posséderaient des mots aux significations opposées comme «bon et " mauvais ", " haut » et "bas ». Freud essaye d'expliquer ces phénomènes étranges en disant que dans les langues archaïques, les processus inconscients fonctionnaient comme dans le rêve ou le lapsus. Or, la "condensation » de deux sens opposés, ce mécanisme propre au travail du rêve ne serait-il pas explicable par la théorie hjelmslévienne ? Car par le syncrétisme d'un terme intensif et d'un terme extensif, l'opposition est supprimée pour obtenir un terme extensif ${ }^{10}$. Je me demande donc si cette théorie radicalement nouvelle de la relation n'est pas capable de décrire l'univers de la sublogique ou de la subjectivité consciente-préconsciente-inconsciente, où les identités seraient instables et où " tout serait soi et autre que soi, donc ni soi ni autre ${ }^{11}$ ? On sait qu'Émile Benveniste était incapable de se rendre compte de la portée de la réflexion freudienne sur le sens opposé, sans doute à cause de sa conception étroite du système: la langue serait un système logicomathématique fondé sur le principe de contradiction; tandis que la théorie de Hjelmslev semble avoir une très grande portée et permettre d'inclure les connaissances apportées par la « psychologie des profondeurs ( Freud, Adler, Jung, etc.). 
16 Autrement dit, Hjelmslev n'a pas besoin de la notion de discours pour étudier la subjectivité dans le langage: la notion de langue lui suffit. C'est là, me semble-t-il, la grande différence entre le structuralisme danois d'une part et le structuralisme pragois et parisien d'autre part ${ }^{12}$.

\section{Les anagrammes}

Les mots dans les Anagrammes de Saussure échappent à la logique de la science dominée par le principe de contradiction, car on sait que ces «mots sous les mots» jouent comme à la fois $A$ et non-A. On peut donc dire qu'ils jouent selon la logique des paragrammes pour employer l'expression de Julia Kristeva. Or, la logique des paragrammes, caractérisée par la co-présence de termes contradictoires, n'est-elle pas identique à cette prélogique, une des composante de la sublogique hjelmslévienne? On a vu que la langue est un système sublogique qui est à la fois logique et prélogique, donc à la fois logique scientifique et logique paragrammatique. Si Hjelmslev a mis en évidence le fait que la langue est un système sublogique d'oppositions participatives, ne pourrait on pas penser qu'il nous suggère également pourquoi la production des anagrammes ou du langage poétique est possible dans la langue? Dans l'optique hjelmslévienne, les anagrammes ne seraient jamais une déviance pure et simple de la langue normative : ce sont, au contraire, les potentialités fécondes de la langue, ou la logique scandaleuse propre à la langue qui permettent de produire des anagrammes ou du langage poétique.

Hjelmslev, mort en 1965, n'était sans doute pas au courant de l'existence des notes de Saussure sur les Anagrammes. Mais peu importe. Ces anagrammes que Saussure ne pouvait pas comprendre peuvent être expliqués formellement par la théorie de Hjelmslev. Les anagrammes, rejetés par Saussure à l'extérieur de la linguistique, peuvent être récupérés dans l'objet même de la linguistique : dans la langue ${ }^{13}$.

\section{Hjelmslev, cet inconnu}

Ainsi, Hjelmslev, qui est parti du Saussure du Mémoire et du Cours, arrive à rencontrer paradoxalement le troisième Saussure, celui des Anagrammes. On s'aperçoit que l'originalité de Hjelmslev consiste à réaliser le développement et le dépassement de Saussure par l'unique moyen de l'immanentisme. Il réussit à récupérer dans la langue tout ce que Saussure a rejeté, sans doute malgré lui, au-dehors de la linguistique : le sujet parlant, le dynamisme des forces et les anagrammes. Le structuralisme de Hjelmslev, dynamique et moniste, semble donc ouvrir la possibilité de formaliser tout cela à l'intérieur même de la théorie du langage.

Tout ce parcours, si paradoxal soit-il, semble démontrer que Hjelmslev est le véritable et unique continuateur de Saussure et que ce brillant saussurien danois reste encore un grand inconnu ${ }^{14}$. 


\section{NOTES}

1. Voir Knud Togeby, Structure immanante de la langue française, Larousse, Paris, 1965, pp. 5-6.

2. Louis Hjelmslev, «Linguistique structurale» (1948), Essais linguistiques, Travaux du Cercle Linguistique de Copenhague, vol. I, 11, Sprog- og Kulturforlag, Copenhauge, 1970, p. 21.

3. Voir Kenji Tatsukawa, "Chikara» no shiso-ka Saussure (Saussure penseur des " forces »), Shoshi Kaze no bara, Tokyo, l'986; Kenji Tatsukawa, «Du sens : Le (post-)saussurisme et son autre», Histoire Epistémologie Langage, Tome 11 - fascicule II, Presses universitaires de Vincennes, Paris, 1989.

4. Louis Hjelmslev, Principes de grammaire générale, Det Kgl. Danske Videnskabernes Selskab, Copenhague, 1928, p. 56. Je souligne.

5. Louis Hjelmslev, « Pour une sémantique structurale » (1957), Essais linguistiques, op. cit., p. 97.

6. Il a déjà été question dans ce colloque de cette position d'entendre chez Saussure, et c'est aussi un point de vue que j'ai développé dans mes travaux sur Saussure. Voir la note (3).

7. Louis Hjelmslev, La catégorie des cas, Étude de grammaire générale, Universitetsforlaget i Aarhus, 1935-37 ; réédition, Wilhelm Fink Verlag, Munchen, 1972, p. 37. Je souligne.

8. Ibid. P. 102.

9. Freud, L'Inquiétante étrangeté et autres essais, Traduit de l'allemand par Bertrand Féron, Gallimard, Paris, 1985, pp. 47-60.

10. Voici un exemple trés simple du syncrétisme qu'on trouve en anglais : ainsi l'opposition entre young, terme intensif (區), et old, terme extensif ( ) :

- Mr. P. is old. / His wife is young.

Mais, par le syncrétisme, on obtient :

- How old is she?

11. Émile Benveniste, "Remarques sur la fonction du langage dans la découverte freudienne » (1956), Problèmes de linguistique générale I, Gallimard, Paris, 1966, p. 83.

12. J'engloberais dans le structuralisme danois non seulement Hjelmslev mais aussi Viggo Brøndal. On sait que ces deux linguistes danois étaient en conflit sur le plan aussi bien scientifique que personnel et que leur position théorique était très différente : grosso modo, le structuralisme de Brøndal est transcendentaliste par opposition à l'immanentisme de Hjelmslev. Il me semble néanmoins que ces deux positions peuvent s'unir pour constituer un seul structuralisme danois contre le structuralisme pragois et parisien. Pour les détails, voir Kenji Tatsukawa et Hiroaki Yamada, Gendai Gengo- ron, Saussure, Freud, Wittgenstein (Théories contemporaines du langage, Saussure, Freud, Wittgenstein), Shinyo-sha, Tokyo, 1990 ; Kenji Tatsukawa, Yuwaku-ron, Gengo to (shiteno) shutai (Théorie de la séduction, Le sujet e(s)t le langage), Shinyo-sha, Tokyo, 1991.

13. Nicolas Ruwet, qu'on pourrait considérer comme un représentant de la linguistique orthodoxe, reproche à Hjelmslev d'élaborer une théorie linguistique dont les applications ne seraient pas du côté du langage ordinaire, mais du côté du « Jabberwocky » de Lewis Carroll ou de Finnegans Wake de James Joyce, c'est-à-dire du côté du langage littéraire (Introduction à la grammaire générative, Plon, Paris, 1967, p. 54).

14. Il va sans dire que Hjelmslev n'est pas du tout un «inconnu » pour des sémioticiens autour d'A.-J. Greimas, dont quelques-uns sont présents dans ce colloque; et moi-même, dans mes réflexions sur ce linguiste danois, je me suis souvent référé à leurs travaux.

J'estime que les travaux de Claude Zilberberg entre autres ont une importance décisive pour 
l'étude hjelmslévienne actuelle. Voir par exemple, son livre Raison et poétique du sens, P.U.F., Paris, 1988. 